\title{
Feasibility of High Spatial Resolution Working Modes for Clinical PET Scanner
}

\author{
Kelin Wang \\ Department of Radiation Oncology, Penn State Hershey Medical Center, Hershey, PA, USA \\ Email: KWang1@pennstatehealth.psu.edu
}

How to cite this paper: Wang, K. (2018) Feasibility of High Spatial Resolution Working Modes for Clinical PET Scanner. International Journal of Medical Physics, Clinical Engineering and Radiation Oncology, 7, 539-552.

https://doi.org/10.4236/ijmpcero.2018.74045

Received: October 24, 2018

Accepted: November 25, 2018

Published: November 28, 2018

Copyright $\odot 2018$ by author and Scientific Research Publishing Inc. This work is licensed under the Creative Commons Attribution International License (CC BY 4.0).

http://creativecommons.org/licenses/by/4.0/

\begin{abstract}
Contemporary PET scanners for clinical use have spatial-resolution of 4 - 5 $\mathrm{mm}$, caused by fundamental factors in medical imaging: detector sizes, free path of positrons, and non-colinearity uncertainty of annihilation photon-pairs. The drawback in resolution significantly restrained the sensitivity of PET in imaging small lesions, which could be either early-stage cancers or small metastasis. In this study, the principle for a novel scanning mode to acquire high spatial-resolution images is proposed for clinical PET scanners. The concept of equivalent position was first proposed as different angular orientations of the scanner ring, at which comparable images could be achieved. Due to this concept, a typical static PET scan can be separated into $m(m \geq 2)$ equivalent sub-scans at different equivalent positions, when the scanner ring is systematically adjusted to $m$ equivalent-positions of equal distance within one detector size. In this case each detector is virtually divided into $m$ equal sub-detectors, without physical minimizing the detector size, and imaging contributions from every $1 / \mathrm{m}$ part of the detector can be determined by an analytically matrix, since there are $m$ variables and $m$ sub-scans. This novel concept is quite feasible to contemporary design because the high spatial resolution working modes $(m \geq 2)$ only demand the scanner to be slightly adjustable to other angular orientations. Adding high spatial resolutions modes to the scanner only has trifling influence on contrast resolutions as all imaging events at each sub-scan are independent. The time for performing a high-resolution scan could be comparable to a typical PET scan, as long as the Poisson noises are insignificant to low-uptake voxels. As a result, for a typical scanner design e.g. $80 \mathrm{~cm}$ in diameter with ${ }^{18} F$ as tracers, the spatial resolution of double sub-scans $(m=2)$ is $2.56 \mathrm{~mm}$, and $2.19 \mathrm{~mm}$ for triple sub-scans $(m=3)$, which are significant improvements. The novelty of high spatial resolution design is compatible to digital PET or any other technological evolutions.
\end{abstract}




\section{Keywords}

PET Scanner, High Spatial Resolution, Equivalent Imaging Position

\section{Introduction}

Clinical positron emission tomography (PET) imaging has become indispensable in cardiology and cancer diagnosis since 1990s [1]-[10], largely due to the technological evolution for the combined unit of PET-CT [11]. In favor of PET radiobiological contrast, similar combined unit of MR-PET was recently developed, though enhancing PET image quality with MRI information remained a challenging topic [12] [13]. Nevertheless, poor spatial resolution is deemed a drawback in clinical PET imaging because some clinical factors, e.g. reasonable diameter of scanner, must be considered in design hence inevitably drop the spatial resolution. As known, early-stage cancers could be successfully controlled if they are diagnosed on time. However, this has been tremendously challenging because small tumors in PET were blurred with low-uptake surrounding tissues in any given voxel due to resolution limit [14]. In many situations, the best time for treatment was already missed when the tumor could be possibly imaged with clinical PET-CT. Similarly, cancer metastasis could not be imaged earlier either by PET-CT due to the limitation of spatial resolution.

Spatial resolution of PET (denoted by $R$ ) is limited by multiple components, either solvable or fundamental [15] [16] [17] [18]. Solvable components are essentially caused by PET scanner engineering, such as decoding uncertainty caused by less electronic channels for detectors [15]; penetrating uncertainty caused by the $\gamma$-photons missing the detector surface [16]; and sampling uncertainty caused by in homogeneity of sampling probability in scanner field of view [16]. All solvable components could be ultimately removed or significantly minimized in engineering, either by optimizing hardware or by enhancing reconstruction algorithm [17] [18]. Fundamental components are essentially set by nature, which include 3 terms: range of positron (denoted by $R_{R}$ ) in tissues [19] [20] [21]; non-colinearity uncertainty (denoted by $R_{N C}$ ) of positronium annihilations [22]; and the detector size.

The range of positron $R_{R}$, (FWHM of range profile) is the mean distance between the radionuclide (where the $\beta^{+}$decay occurred) and the position where the positronium annihilated, thus $R_{R}$ depends on tissue densities and radionuclide types i.e. the energy of positrons. For ${ }^{18} F, R_{R}=0.54 \mathrm{~mm}$ in water for endpoint energy of $0.64 \mathrm{MeV}$ [23].

In contemporary PET design, scintillating signals from detectors were collected and amplified by photomultiplier tubes (PMTs) into analog signals, rather than directly converted into digital signals. For discrete detectors of size $d$, the detector resolution is half of the detector size $d / 2$.

Either $R_{R}$ or the detector size is more lucid than $R_{N O}$ since $R_{N C}$ was not discovered 
until people learned that $R$ actually became worse if one enlarged scanner diameter to achieve better resolution. $R_{N C}$ accounts for the non-zero net-momentum of positronium in the center-of-mass frame of reference. In another words, a positronium is in thermal motion like other molecules at body temperature, causing uncertainty in exit directions for annihilation photons. Obviously, cooling the patient would help since that reduced thermal motions for all particles in body; however, this would also tremendously alter the physiological processes (e.g. glucose metabolism) to be imaged in PET [22]. For this reason $R_{N C}$ is also considered fundamental. In practical, $R_{N C}$ accounts for non-colinearity caused by pair-photon annihilations only, though a positronium could annihilate through either pair-photons (back-to-back) or triplet-photons (apart by 120 degrees) with almost 50\% probabilities each [24] [25] [26]. As known, the PET spectrum contains a distinctive peak at $511 \mathrm{KeV}$ formed by pair-photons through photoelectric effect (PE), and a broad low-energy spectrum mainly caused by Compton scattering. Detecting triplet-photons PE events is difficult because signals from those photons overlapped with the Compton noises. Consequently, at body temperature, $R_{N C}$ can be approximately expressed as a constant times the diameter $D$ (in unit of $\mathrm{mm}$ ) of the scanner:

$$
R_{N C}=0.0022 \times D
$$

Although $R_{N C}$ is proportional to scanner diameter, shrinking scanner size for better $R_{N C}$ is not an option due to clinical requirement. The only exception might be the micro-PET scanners for small animal studies, which typically contains a compact scanner of $10-15 \mathrm{~cm}$ in diameter, and this leads to $R \approx 1 \mathrm{~mm}$ [27]. Following the NEMA NU2-2012 standard for regulations in spatial resolution, sensitivity, count rate curve and image quality (IQ) parameters [28], a clinical PET scanner demands a diameter of at least $80 \mathrm{~cm}$ to scan most of the patients, and the detector size is typical $7-10 \mathrm{~mm}$ to have a reasonable efficiency, which gives rise to $R$ of $4-5 \mathrm{~mm}$.

The overall spatial resolution can be expressed as:

$$
R=\sqrt{R_{R}^{2}+R_{N C}^{2}+(d / 2)^{2}}
$$

Since neither $R_{R}$ nor $R_{N C}$ could be possibly optimized in design, more efforts were put into detector resolution to improve whole spatial resolution. The choice of detector size is also a clinical consideration, because using smaller detectors causes low collecting efficiency, hence requiring more injection radionuclides/dose to the patients. In recent development of digital-PET, the analog PMTs in the traditional design were replaced by solid-state avalanche photodiodes (APD) coupled by digital acquisition system [29] [30] [31], which improved collecting efficiency as well as spatial resolution [32]. Suppose the spatial resolution with APD was much better than $d / 2$, replacing the third term in Equation (2) with $R_{R D E T}^{2}$ representing the spatial resolution by detector, Equation (2) can be re-written as:

$$
R=\sqrt{R_{R}^{2}+R_{N C}^{2}+R_{D E T}^{2}}
$$


As reported, $R_{D E T}<d / 2$, the technological evolution of digital PET would certainly improve $R$, although more convincing results are expected in the future.

This feasibility study also attempted to improve detector response for better spatial resolution, as a parallel approach to digital-PET. Other than directly digitizing the scintillating signals, this study focused on high resolution working modes for clinical PET scanner, without significantly altering prevalent clinical PET design. Implementing high resolution working modes is compatible to any parallel technological evolution such as digital-PET, and could be potentially meaningful to any other emission-based tomography.

\section{Methods and Materials}

\subsection{Equivalent Positions for Imaging}

Suppose 2 clinical PET-scanners of the same type were manufactured, with each consisting of $N$ identical detectors of size $\rho$ as defined below, but the orientations of the detectors are slightly different in those 2 scanners. As shown in Figure 1, the angular positions of $N$ detectors in the first scanner are $\Theta_{A}=\left\{\theta_{1}, \theta_{2}, \ldots, \theta_{N}\right\}$; and in the second scanner all detectors are rotated to a clockwise angle $\varepsilon$ accordingly, thus the angular positions become $\Theta_{B}=\Theta_{A}+\mathcal{E}=\left\{\theta_{1}+\mathcal{\varepsilon}, \theta_{2}+\mathcal{\varepsilon}, \ldots, \theta_{N}+\right.$ $\varepsilon$. If both scanners are used to scan the same patient at identical imaging conditions, obviously, there is no significant difference between those 2 PET scans as long as sufficient events are collected. For instance, they should have the same contrast and spatial resolution, thus the two imaging results are equivalent.

Equivalent-positions are defined as positions of different orientation for the detector ring that can generate equivalent images (Figure 1). This concept is valid only when statistically large amounts of events are reconstructed into an image. For above case, $\Theta_{A}$ and $\Theta_{B}$ are examples of 2 equivalent-positions.

\subsection{Optimal Detector Size}

For a typical PET scan using ${ }^{18} \mathrm{FDG}$ tracer, the theoretical limit of spatial resolution would be $1.8 \mathrm{~mm}$ for an $80 \mathrm{~cm}$-diameter scanner, if the detector size were minimal $\left(R_{D E T}=0\right)$. In contemporary design, the selection of detector size is of a
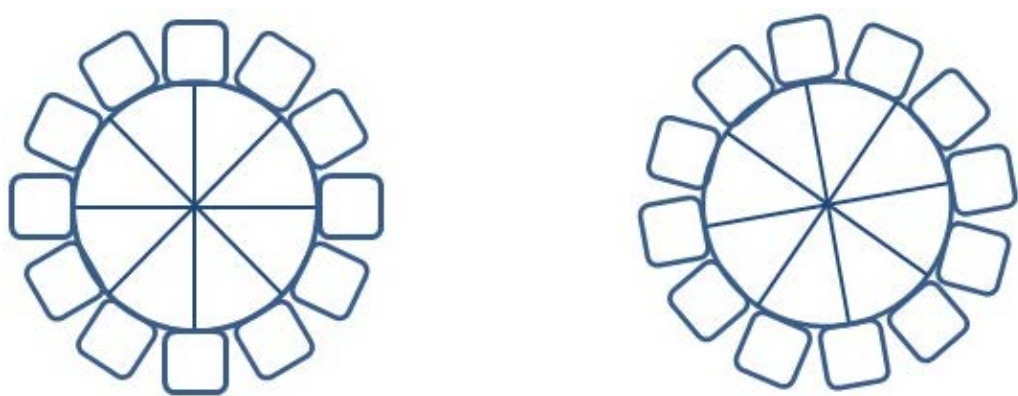

Figure 1. Schematic drawing for 2 equivalent imaging positions of the same PET scanner composed of 12 detectors. The difference between the left and the right position is that the right one rotates a small degree of half detector size. The images acquired from those 2 positions are equivalent if there is sufficient number of detectors. 
major concern on collecting efficiency, which also depends on engineering and electronic limits, e.g. collimator, deadtime, energy resolution etc. However, for any successful emission-based tomography, there always exists an optimal detector size, as a result of compromising collecting efficiency and spatial resolution. With any advancement of technological evolution such as digital-PET, the optimal detector size might also change. The mathematical relationship between optimal detector size and spatial resolution will be discussed elsewhere.

Since all PET scanners are cylindrical, it would be convenient to use angular size rather than physical size of detector. The angular size $\varphi$ of each detector in a ring is: $\varphi=\left|\theta_{2}-\theta_{1}\right|=\left|\theta_{3}-\theta_{2}\right|=\ldots=\left|\theta_{N}-\theta_{N-1}\right|=2 \pi / N$, and the optimal size of detector can be expressed in term of angular size: $\rho=(D / 2) \varphi$, in which $D$ is the diameter of the scanner.

\subsection{Spatial Resolution Enhancement with Imaging at 2 Equivalent-Positions}

For a PET scanner ring of $N$ detectors, a PET image (denoted by $F$ ) is virtually the superposition of sub-images contributed by $N$ individual detectors. However, remember that any event in PET imaging is a coincidence of 2 detectors, thus counting the events at each detector actually doubling the counts in statistics. Nevertheless, for convenience a PET image $F$ can still be represented by superposition of $N$ sub-images contributed by $N$ individual detectors, but mathematically counted twice:

$$
F=\frac{1}{2} \sum_{i=1}^{N} f_{i}
$$

The 1/2 at Equation (3) means the events were counted twice after checking all $N$ detectors. Assume an adjustable PET scanner takes 2 images (at identical imaging conditions) $F_{A}$ and $F_{B}$ at 2 equivalent-positions $\Theta_{A}$ and $\Theta_{B}$, respectively. Re-write Equation (3) for each equivalent-position respectively, sum up those 2 equations and then divide by 2 for each side. Given that $F$ is the same for $2 \mathrm{im}$ ages $\left(F_{A}=F_{B}=F\right)$, we have:

$$
F=\frac{1}{4} \sum_{i=1}^{N}\left(f_{i}^{(A)}+f_{i}^{(B)}\right)
$$

In which $f_{i}^{(A)}$ and $f_{i}^{(B)}$ stand for the sub-images of the $I^{\text {th }}$ detector at $\Theta_{A}$ and $\Theta_{B}$ respectively.

Consider the simplest situation, in which the orientation difference between those 2 equivalent-positions is exactly half of the angular size of detectors $\varphi$, i.e. $\Theta_{B}=\Theta_{A}+\varepsilon=\Theta_{A}+\varphi / 2$ (Figure 2), both $f_{i}^{(A)}$ and $f_{i}^{(B)}$ can be represented by 2 analytical terms respectively, and

$$
\begin{aligned}
& f_{i}^{(A)}=f_{i, 1}+f_{i, 2} \\
& f_{i}^{(B)}=f_{i-1,2}+f_{i, 1}
\end{aligned}
$$

Note $f_{N+1,2}=f_{1,2}$ Equation (4) becomes: 


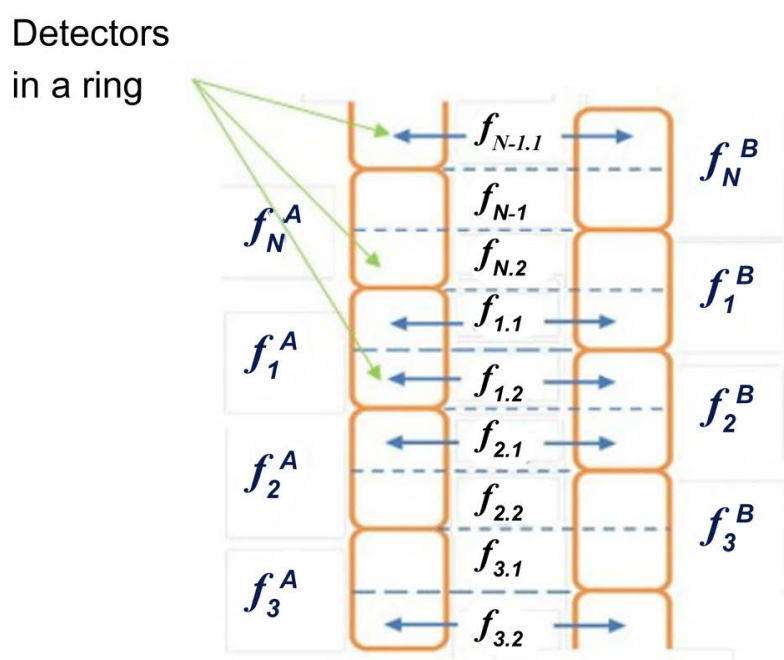

Equivalent positions

Figure 2. Schematic drawing for $2(m=2)$ equivalent imaging positions. There are $N$ scintillator detectors in a scanner ring, so detector No. 1 and No. $N$ are adjacent to each other. The left side is for equivalent-position $A$ and the right is for $B$, respectively. Equivalent-position $B$ is a small adjustment (after a small rotation of $\varphi / 2$ ) from position $A$, thus each detector in position $B$ overlaps with 2 detectors in position $A$ (exact half for each detector). Since imaging at $A$ and $B$ are equivalent, the 2 sub-images contributed by each detector can analytical to image manipulations/calculations.

$$
F=\frac{1}{4} \sum_{i=1}^{N}\left(f_{i}^{(A)}+f_{i}^{(B)}\right)=\frac{1}{2} \sum_{i=1}^{N} \sum_{k=1}^{2} f_{i, k}=\frac{1}{2} \sum_{i=1}^{N}\left(f_{i, 1}+f_{i, 2}\right)
$$

In another word, image $F$ can be represented by $2 N$ sub-images from $2 N$ detectors ("sub-detector"), rather than $N$ sub-images from $N$ detectors. Please note that in Equation (4') both $f_{i, 1}$ and $f_{i, 2}$ still use the same position i.e. $\theta_{\mathrm{i}}$ of detector $i$, even though it is already known that $f_{i, 1}$ is from the lower half and $f_{i, 2}$ is from the upper half of the detector (Figure 2). If both terms are assigned more precise positions, respectively, i.e. for lower half of a given detector $\theta_{i} \rightarrow \theta_{i}-\varphi / 4$ thus $f_{i, 1} \rightarrow f_{i, 1}^{\prime}$; and for upper half of the detector $\theta_{i} \rightarrow \theta_{i}+\varphi / 4$ thus $f_{i, 2} \rightarrow f_{i, 2}^{\prime}$, the new superimposed image $F$ 'should also differ from the original image $F$, because each detector is now separated into 2 pseudo sub-detectors of equal size $\rho / 2$, and each contributes a known sub-image independently. Re-write Equation (4') for the new positions, and re-labeled the terms by $f_{i, 1}^{\prime}=f_{2, j-1}^{\prime}$ and $f_{i, 2}^{\prime}=f_{2, j}^{\prime}$ we have:

$$
F^{\prime}=\frac{1}{2} \sum_{i=1}^{N}\left(f_{i, 1}^{\prime}+f_{i, 2}^{\prime}\right)=\frac{1}{2} \sum_{j=1}^{2 N} f_{j}^{\prime}
$$

Compare Equation (3) and Equation (6), it is obvious to tell that after taking images at 2 equivalent-positions, the original PET image $F$ which was contributed by $N$ detectors of optimal size $\rho$, can be improved to $F$ ' of better quality, contributed by $2 N$ detectors of size $\rho / 2$. The improvement in spatial resolution from single to double equivalent-position is shown in Figure 3 schematically. 

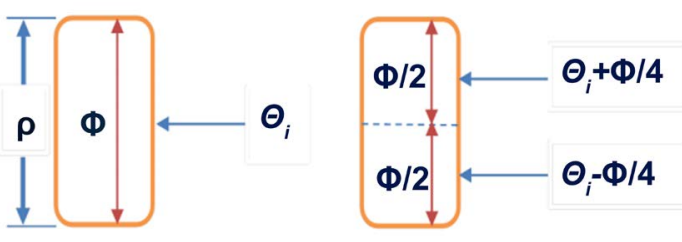

Figure 3. Schematic drawing for spatial resolution improvement. The left drawing illustrates the resolution of normal scans: all events collected by the detector are assumed at position $\theta_{p}$, thus the uncertainty of events positions i.e. the detector size $\rho$ (or angular size $\varphi)$ contributed to the limit of spatial resolution. The right drawing illustrates the resolution of imaging at 2 equivalent-positions: since the sub-images at each half of detector are determined, and each is assigned with a new position i.e. $\theta_{i} \pm \varphi / 4$, the uncertainty of events positions is only half as before, thus the resolution is improved.

Solving the matrix denoted by Equation (5), the sub-images at each sub-detector can be acquired analytically. A modeling algorithm on image manipulation is needed to fulfill this purpose at image/sub-image level, though the detail of modeling algorithms is obviously beyond the scope of this context. Replacing $R_{D E T}$ by $R_{D E T} / 2$ in Equation (2'), a clinical PET-scanner using ${ }^{18} F$ as tracers may improve the spatial resolution from $4 \mathrm{~mm}$ to $2.6 \mathrm{~mm}$ (Table 1 ).

\subsection{Voxel-Based High-Resolution Mode}

The method mentioned above is virtually for pixel-based or 2-dimensional imaging. To generate a 3-dimensional or voxel-based image of better spatial resolution, imaging at 2 longitudinal equivalent-positions is needed, such that the longitudinal spatial resolution is also improved. This could be easily achieved by moving the imaging table for a distance of half detector size, with the same mechanism denoted by Equations (3) to (6).

\subsection{High Spatial Resolution with Imaging at Multiple Equivalent-Positions}

The more general cases are imaging at multiple equivalent positions, as long as the PET scanner is adjustable to those equivalent-positions. Since all detectors are instrumented symmetrically in the scanner ring, the maximal angular displacement to achieve all possible equivalent-positions is virtually $\varphi$, i.e. the size of given detectors. To perform imaging at $m$ equivalent-positions, the scanner needs to adjust its orientation $m-1$ times, with equal angular distances for each adjustment. If $m=1$, the scanner is working at normal mode (at high spatial resolution modes $m>1$ ) and hence there is no need to adjust its orientation.

Similar to imaging at double equivalent positions $(m=2)$, which was discussed at Equation (5) and Equation (6), for imaging at triple equivalent positions $(m=3)$, Equation (5) can be expanded to:

$$
\begin{aligned}
& f_{i}^{(A)}=f_{i, 1}+f_{i, 2}+f_{i, 3} \\
& f_{i}^{(B)}=f_{i-1,3}+f_{i, 1}+f_{i, 2} \\
& f_{i}^{(C)}=f_{i-1,2}+f_{i-1,3}+f_{i, 1}
\end{aligned}
$$


Table 1. Spatial resolution improvements by imaging at multiple equivalent-positions, calculated with Equation (2) and Equation (10) for scanner diameter $D=80 \mathrm{~cm}$. Please note $R_{R}=0.54 \mathrm{~mm}$ is used for ${ }^{18} F$ in the calculations. $R_{0}$ stands for the spatial resolution of contemporary PET scanners (4-5 mm) for typical PET scans, and $R$ stands for the true resolution after imaging at $m$ equivalent-positions, thus $R=R_{0}$ for $m=1$. $R_{0}$ was first assumed with Equation (2) to determine the detector size. $R$ was then calculated.

\begin{tabular}{cccc}
\hline $\begin{array}{c}\text { No. of } m \\
\text { (Equivalent positions) }\end{array}$ & $\mathrm{R}(\mathrm{mm})\left(R_{0}=4.0\right)$ & $R(\mathrm{~mm})\left(R_{0}=4.5\right)$ & $R(\mathrm{~mm})\left(R_{0}=5.0\right)$ \\
\hline 1 & 4.00 & 4.50 & 5.00 \\
2 & 2.56 & 2.76 & 2.97 \\
3 & 2.19 & 2.29 & 2.41 \\
4 & 2.04 & 2.11 & 2.18 \\
5 & 1.97 & 2.02 & 2.06 \\
\hline
\end{tabular}

For imaging at $m$ equivalent-positions, each given detector virtually separates its contributions into $m$ sub-detectors of known positions determined by those equivalent-positions. In this case $m \times N$ variables need to be solved if there are $N$ detectors, however, these are always analytical because images were taken at $m$ equivalent-positions and hence there have been $m \times N$ readouts. In a more general form of imaging at $m$ equivalent-positions, Equation (4) can be re-written as:

$$
F=\frac{1}{2 m} \sum_{i=1}^{N} \sum_{k=1}^{m} f_{i}^{(k)}
$$

In Equation (7) $f_{i}^{(k)}$ is the sub-image of the $i^{\text {th }}$ detector $(i=1,2, \ldots, N)$ imaging at the $k^{t h}(k=1,2, \ldots, m)$ equivalent-position, and similar to Equation (5) $f_{i}^{(k)}$ can be represented by $m$ terms:

$$
\left(\begin{array}{c}
f_{i}^{(1)} \\
f_{i}^{(2)} \\
\cdots \\
f_{i}^{(m-1)} \\
f_{i}^{(m)}
\end{array}\right)=\left(\begin{array}{c}
f_{i, 1}+f_{i, 2}+f_{i, 3} \ldots+f_{i, m-1}+f_{i, m} \\
f_{i-1, m}+f_{i, 1}+f_{i, 2} \ldots+f_{i, m-2}+f_{i, m-1} \\
\ldots \\
f_{i-1,3}+f_{i-1,4}+\ldots+f_{i, 1}+f_{i, 2} \\
f_{i-1,2}+f_{i-1,3}+\ldots+f_{i-1, m}+f_{i, 1}
\end{array}\right)
$$

Plug in Equation (8) into Equation (7), and re-label the items, i.e. $f_{i, m} \rightarrow f_{m, j}^{\prime}, \quad f_{i, m-1} \rightarrow f_{m, j-1}^{\prime}, \ldots$ etc., we have:

$$
F^{\prime}=\frac{1}{2} \sum_{j=1}^{m N} f_{j}^{\prime}
$$

Similar to Equation (6) for imaging at double equivalent positions, Equation (9) indicates that a PET scan $F$ can be represented by $m \times N$ sub-images from $m$ $\times N$ pseudo sub-detectors. Re-write Equation (2'), the overall spatial resolution for imaging at $m$ equivalent-positions is:

$$
R=\sqrt{R_{R}^{2}+R_{N C}^{2}+\left(R_{D E T} / m\right)^{2}}
$$

Equation (10) shrinks to Equation (2') if $m=1$, which is for the normal 
working mode. The theoretical best spatial resolution $\left(R=1.8 \mathrm{~mm}\right.$ for $\left.{ }^{18} F\right)$ occurs when $m \rightarrow \infty$ such that $R_{D E T} / m \rightarrow 0$. However, imaging at many equivalent-positions cannot improve the spatial resolution too much. Table 1 listed the improvements of spatial resolution as a function of $m$ for a contemporary scanner of diameter $80 \mathrm{~cm}$. The greatest improvement occurs when $m$ changed from 1 to 2 , thus $m=2$ is preferably recommended.

Since the spatial resolution could be improved this way, it would be possible to design relatively larger scanners only by slightly reducing the spatial resolution. Larger scanners have the advantage of scanning patients of larger in size. Figure 4 illustrate the spatial resolution as a function of $m$ for detector size $d$ equals to $8 \mathrm{~mm}, 9 \mathrm{~mm}$ and $10 \mathrm{~mm}$, for scanner diameter $D=80 \mathrm{~cm}$. Figure 5 and Figure 6 are virtually similar plots for other scanner sizes, i.e. $D=90 \mathrm{~cm}$ and $100 \mathrm{~cm}$, respectively. All figures represent the same result that at the spatial resolution improves most significantly from $m=1$ to $m=2$.

\section{Results and Discussions}

\subsection{Procedure to Take PET Scans at High Spatial Resolution Modes}

The pre-scan procedure for taking high-resolution scans is virtually identical to that for typical PET scans. Suppose a high-resolution mode (e.g. $m=2$ ) is chosen, time slots for 2 sub-scans are assigned to 2 equivalent-positions by the system with consideration the trace decays, and the sub-scans at each equivalent-position are taken sequentially. The equivalent-positions should be evenly distributed within the angular size $\varphi$ to ensure the precision of sub-detector size.

Since the PET scanner is cylindrically symmetric, equivalent positions at longitudinal direction are independent to equivalent positions at angular direction. With this concern, the total sub-scans at $m$ equivalent-positions at both angular

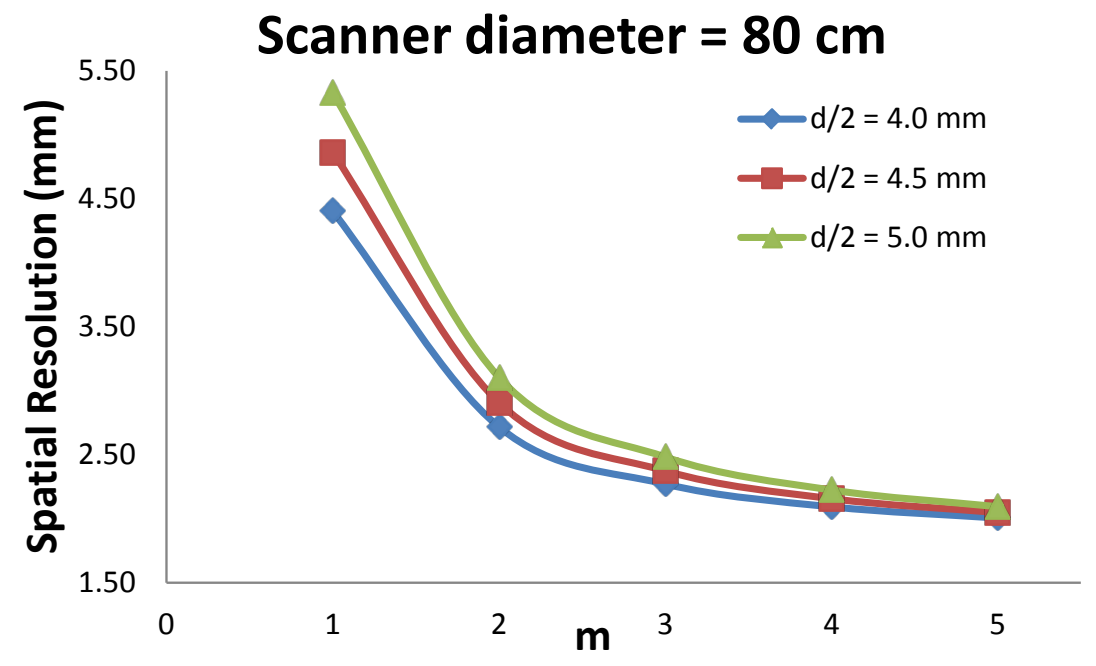

Figure 4. For a scanner of $80 \mathrm{~cm}$ in diameter, the distribution of spatial resolution vs the $m$ value for different detector sizes. Note the detector resolution is $d / 2$ if $d$ is the detector size. 


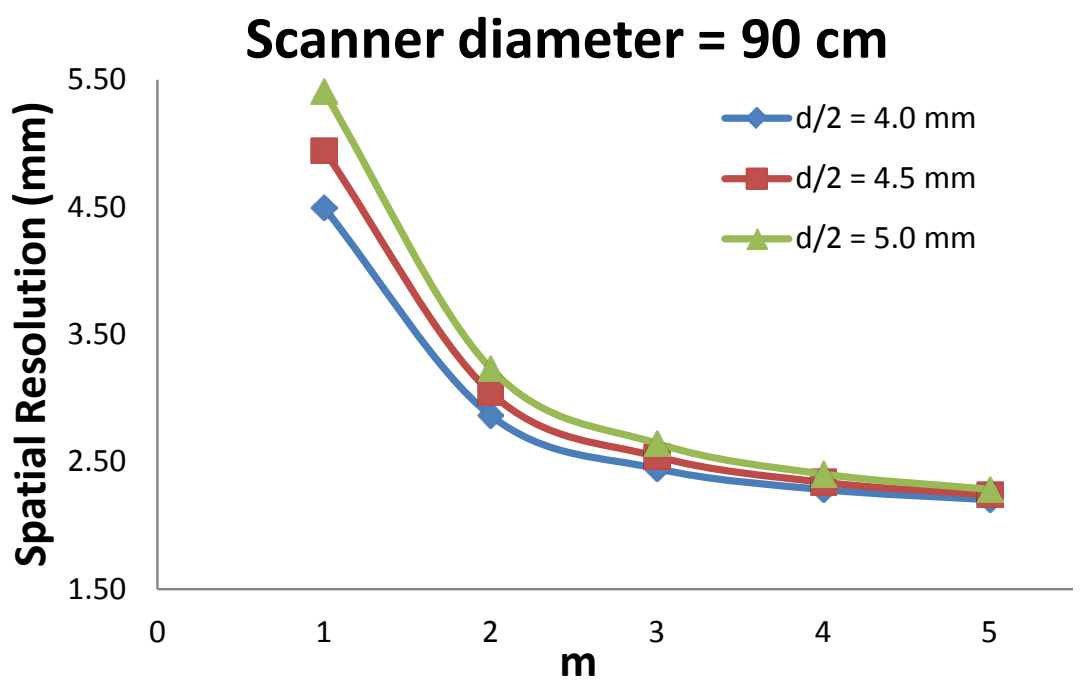

Figure 5. For a scanner of $90 \mathrm{~cm}$ in diameter, the distribution of spatial resolution vs the $m$ value for different detector sizes. Note the detector resolution is $d / 2$ if $d$ is the detector size.

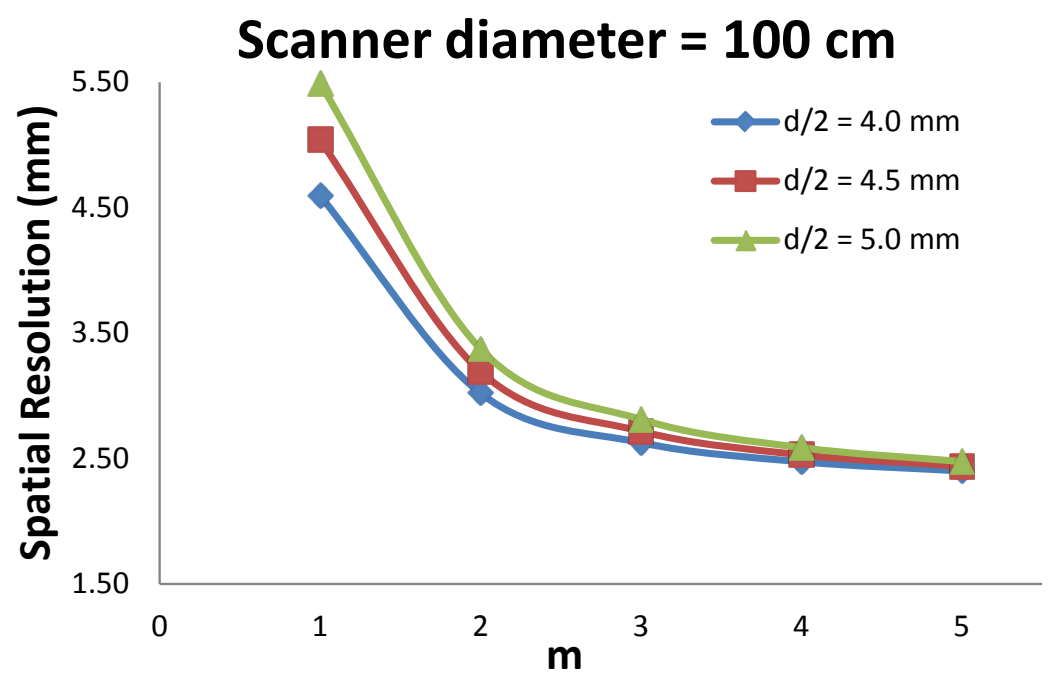

Figure 6. For a scanner of $100 \mathrm{~cm}$ in diameter, the distribution of spatial resolution vs the $m$ value for different detector sizes. Note the detector resolution is $d / 2$ if $d$ is the detector size.

and longitudinal directions would be $m^{2}$ times (e.g. $m^{2}=4$ if $m=2$ is chosen) as normal working mode.

\subsection{Novelty in Scanner Design}

Unlike CT units, traditional PET scanners were stationary and not adjustable to different orientations. Working at high resolution modes also requests the scanner to be stationary while taking the high resolution PET scans. However, it also requires the scanner to be adjustable to different angular equivalent positions. This would slightly alter the traditional PET scanner design by adding the rotational capability for a maximal distance of 1 detector size. 


\subsection{Limitation on Dynamic Imaging}

The essential premise for applying multi-equivalent-position imaging is that positron emissions are stable, which typically occurred after some uptake time e.g. $45 \mathrm{~min}$. This premise actually has been taken for all static PET imaging. Before taking a static PET scan, the patient is normally sent to the waiting room for 45 60 minutes after injecting the tracer e.g. ${ }^{18} \mathrm{FDG}$, and this waiting period ensures sufficient uptakes in the targets. Imaging at 45 minutes or 60 minutes after injection are normally assumed equivalent, since the uptake process has already completed thus there is no need to consider time-dependent uptakes.

Dynamic imaging sometimes is needed, mostly for research purposes. For instance, to discriminate necrosis from hypoxic volumes by the timing responses to the tracers, a dynamic PET scan rather than static PET is normally taken, from the injection moment up to at least 1 hour after injection. In another words, the goal of a dynamic scan is to image the whole uptake process, in which the emission rate for the targets is time-dependent, i.e. changing sharply before it eventually stabilizes. Imaging at high resolution working modes is virtually a special type of static PET imaging, and hence has drawbacks under this scenario. If all sub-images become time dependent, i.e. $f_{i}^{(A)} \rightarrow f_{i}^{(A)}\left(t_{1}\right)$ and $f_{i}^{(B)} \rightarrow f_{i}^{(B)}\left(t_{2}\right)$, the contributions from sub-detectors are no longer analytical. The difficulties of applying high spatial resolution working modes to dynamic imaging are still unclear, although perspective studies are expected for the needs of establishing successful analytical methods.

\subsection{Impact on Contrast Resolution}

A clinical PET scan typically takes 15 - 20 minutes. Imaging at high spatial resolution modes might demand extended scan time, because in each sub-image at equivalent positions the signal to noise ratio might drop since the number of events drop to $1 / \mathrm{m}$ whereas the noise level remains unchanged.

Another major concern would be the Poisson noise for some low-uptake voxels in a given sub-scan at an equivalent-position, which could be avoid by (a) increasing the imaging time at each equivalent-position; or (b) increase the injection amount of tracers to the patient. Option (a) is feasible as long as patient immobilization during a moderately longer scan is properly handled. In addition, organ motions with time should also be considered during longer time. A suitable scan time should be a compromise of both minimizing the Poisson noise and avoiding unnecessary motion artifacts. Option (b) is easier however generally not recommended since it increases the patient exposure, unless for some critical situations when benefit to risk analysis is carefully performed.

\section{Conclusions}

For some indispensable clinical demands, such as the minimal scanner size to accommodate most patients, and the minimal detector size to keep a reasonable collecting efficiency to avoid too much patient dose, prevalent clinical PET scanner 
seems to reach its limit on spatial resolution around $4-5 \mathrm{~mm}$. Since neither the free-path uncertainty nor the non-colinearity uncertainty could be possibly optimized in clinical PET scanner, the only breakthrough seems to be the efforts on detector resolution. Other than technological evolution such as digital PET, imaging at high spatial resolution working modes is another parallel approach which provides great potential on PET advancement.

In this study, the concept of equivalent position of imaging was proposed for the first time. Imaging at those positions are deemed equivalent. Implementing this concept in imaging and reconstruction, a static PET scan could be considered as superposition of equivalent scans at different angular orientations without varying imaging quality and radiobiological information. It could be concluded in theory that high spatial resolution working modes for PET scanners are feasible at imaging manipulation level, after imaging at more than one equivalent imaging position and analyzing the contribution of sub-detectors with more precise positions. The improvement in spatial resolution is significant at $m=2$ (or double-imaging) mode although imaging at multiple equivalent positions $(m>$ 2) would enhance the resolution more. For typical detector size of $7-10 \mathrm{~mm}$, the spatial resolution at $m=2$ could become $2.6-3.0 \mathrm{~mm}$ from 4.0 to $5.0 \mathrm{~mm}$. For this reason, imaging at double equivalent positions is comparatively recommended.

Under high spatial resolution modes, a static PET scan would be capable of imaging much smaller lesions, which is significant on imaging tumors at early stages, and hence greatly improve the opportunities on cancer controls.

\section{Acknowledgements}

The author would gratefully acknowledge Dr. Mutian Zhang for his helpful comments on the development of digital PET scanner.

\section{Funding}

There is no source of funding for this study.

\section{Involved}

There were no human subjects or animal subjects involved in this study.

\section{Conflicts of Interest}

There is no conflict of interests.

\section{References}

[1] Amit, A., et al. (2013) FDG PET/CT in Monitoring Response to Treatment in Gynecological Malignancies. Current Opinion in Obstetrics and Gynecology, 25 17-22. https://doi.org/10.1097/GCO.0b013e32835a7e96

[2] Dalla Palma, M., et al. (2012) PET/CT Imaging in Gynecologic Malignancies: A Critical Overview of Its Clinical Impact and Our Retrospective Single Center Analysis. Critical Reviews in Oncology/Hematology, 83, 84-98. 
https://doi.org/10.1016/j.critrevonc.2011.10.002

[3] Chen, K., et al. (2011) Positron Emission Tomography Imaging of Cancer Biology, Current Status and Future Prospects. Seminars in Oncology, 38, 70-86.

https://doi.org/10.1053/j.seminoncol.2010.11.005

[4] Lammering, G., et al. (2010) The Use of FDG-PET to Target Tumor by Radiotherapy. Strahlentherapie und Onkologie, 186, 471-481. https://doi.org/10.1007/s00066-010-2150-1

[5] Nestle, U., et al. (2009) Biological Imaging in Radiation Therapy: Role of Positron Emission Tomography. Physics in Medicine \& Biology, 54, R1-R25. https://doi.org/10.1088/0031-9155/54/1/R01

[6] Takahashi, N., et al. (2007) The Roles of PET and PET/CT in the Diagnosis and Management of Prostate Cancer. Oncology, 72, 226-233. https://doi.org/10.1159/000112946

[7] Dimitrakopoulou-Strauss, A., et al. (2006) Quantitative Studies Using Position Emission Tomography (PET) for the Diagnosis and Therapy Planning of Oncological Patients. Hellenic Journal of Nuclear Medicine, 9, 10-21.

[8] Macapinlac, H.A. (2004) FDG PET and PET/CT Imaging in Lymphoma and Melanoma. The Cancer Journal, 10, 262-270. https://doi.org/10.1097/00130404-200407000-00007

[9] Spence, A.M., et al. (2003) Positron Emission Tomography Imaging of Brain Tumors. Neuroimaging Clinics of North America, 13, 717-739. https://doi.org/10.1016/S1052-5149(03)00097-2

[10] Khan, N., et al. (2003) PET in the Follow-Up of Differentiated Thyroid Cancer. British Journal of Radiology, 76, 690-695. https://doi.org/10.1259/bjr/31538331

[11] El Naqa, I., et al. (2007) Concurrent Multimodality Image Segmentation by Active Contours for Radiotherapy Treatment Planning. Medical Physics, 34, 4738-4749. https://doi.org/10.1118/1.2799886

[12] Yoo, H.J., et al. (2015) Integrated Whole Body MR/PET: Where Are We. Korean Journal of Radiology, 16, 32-49. https://doi.org/10.3348/kjr.2015.16.1.32

[13] An, H.J., et al. (2016) MRI-Based Attenuation Correction for PET/MRI Using Multiphase Level-Set Method. Journal of Nuclear Medicine, 57, 587-593. https://doi.org/10.2967/jnumed.115.163550

[14] Schöder, H. (2007) Screening for Cancer with PET and PET/CT: Potential and Limitations. Journal of Nuclear Medicine, 48, 4S-18S.

[15] Moses, W.W., et al. (1993) Empirical Observation on Performance Degradation in Positron Emission Tomographs Utilizing Block Detectors. Journal of Nuclear Medicine, 34, $101 \mathrm{p}$.

[16] Derenzo, S.E., et al. (1981) Imaging Properties of a Positron Tomography with 280 BGO Crystals. IEEE Transactions on Nuclear Science, 28, 81-89. https://doi.org/10.1109/TNS.1981.4331144

[17] De Jong, H.W., et al. (2007) Performance Evaluation of the ECAT HRRT: An LSO-LYSO Double Layer High Resolution, High Sensitivity Scanner. Physics in Medicine \& Biology, 52, 1505-1526. https://doi.org/10.1088/0031-9155/52/5/019

[18] Alessio, A.M., et al. (2006) Modeling and Incorporation of System Response Functions in 3-D Whole Body PET. IEEE Transactions on Medical Imaging, 25, 828-837. https://doi.org/10.1109/TMI.2006.873222

[19] Cho, Z.H., et al. (1975) Positron Ranges Obtained from Biomedically Important Positron-Emitting Radionuclides. Journal of Nuclear Medicine, 16, 1174-1175. 
[20] Derenzo, S.E., et al. (1982) Dynamic Positron-Emission Tomography in Man Using Small Bismuth Germinate Crystals. 6th International Conference on Positron Annihilation, Ft. Worth, 3-7 April 1982, 1-11.

[21] Levin, C.S., et al. (1999) Calculation of Positron Range and Its Effect on the Fundamental Limit of Positron Emission Tomography System Spatial Resolution. Physics in Medicine \& Biology, 44, 781-799. https://doi.org/10.1088/0031-9155/44/3/019

[22] Colombino, P., et al. (1965) Study of Positronium in Water and Ice from 22 to -144 ${ }^{\circ}$ C. Nuovo Cimento, 38, 707-723. https://doi.org/10.1007/BF02748591

[23] Partridge, M., et al. (2006) The Effect of $\beta^{+}$Energy on Performance of a Small PET Camera. Nuclear Instruments and Methods in Physics Research Section A, 568, 933-936. https://doi.org/10.1016/j.nima.2006.09.035

[24] Karshenboim, S.G. (2003) Precision Study of Positronium: Testing Bound State QED Theory. International Journal of Modern Physics A, 19, 3879-3896. https://doi.org/10.1142/S0217751X04020142

[25] Czarnecki, A., et al. (2000) Decays of Positronium. Proceedings of the International Workshop on High Energy Physics and Quantum Field Theory, Vol. 14, Tver, 14-20 September 2000, 538-544.

[26] DeBenedetti, S., et al. (1954) The Three-Photon Annihilation of Positrons and Electrons. Physical Review, 94, 955-959. https://doi.org/10.1103/PhysRev.94.955

[27] Jones, T. and Townsend, D. (2017) History and Future Technique Innovation in Positron Emission Tomography. Journal of Medical Imaging (Bellingham), 4, Article ID: 011013. https://doi.org/10.1117/1.JMI.4.1.011013

[28] Jha, A., et al. (2015) NEMA NU-2 2012 Performance Evaluation of Discovery IQ: A High Sensitivity PET System. Journal of Nuclear Medicine, 56, 1847.

[29] Frach, T., Prescher, G., Degenhardt, C., et al. (2009) The Digital Silicon Photomultiplier-Principle of Operation and Intrinsic Detector Performance. Proceedings of the IEEE Nuclear Science Symposium Conference Record, Orlando, 24 October-1 November 2009, 1959-1965. https://doi.org/10.1109/NSSMIC.2009.5402143

[30] Degenhardt, C., Prescher, G., Frach, T., et al. (2009) The Digital Silicon Photomultiplier-A Novel Sensor for the Detection of Scintillation Light. Proceedings of the IEEE Nuclear Science Symposium Conference Record, Orlando, 24 October-1 November 2009, 2383-2386.

[31] Degenhardt, C., Rodrigues, P., Trindade, A., et al. (2012) Performance Evaluation of a Prototype Positron Emission Tomography Scanner Using Digital Photon Counters (DPC). Proceedings of the IEEE Nuclear Science Symposium and Medical Imaging Conference Record, Anaheim, 27 October-3 November 2012, 2820-2824.

[32] Nguyen, N.C., Vercher-Conejero, J.I., Sattar, A., et al. (2015) Image Quality and Diagnostic Performance of a Digital PET Prototype in Patients with Oncologic Diseases: Initial Experience and Comparison with Analog PET. Journal of Nuclear Medicine, 56, 1378-1385. https://doi.org/10.2967/jnumed.114.148338 